\title{
High Throughput Cryo-Electron Tomography: Towards Molecular Architecture of Spirochetal Flagellar Motor in situ
}

\author{
Jun Liu and Steven J. Norris
}

Department of Pathology and Laboratory Medicine, University of Texas Medical School at Houston, Houston, TX 77030

The bacterial flagellar motor is an extraordinary nanomachine powered by the electrochemical potential of specific ions across the cytoplasmic membrane [1]. The influx of protons through the motor produces either counterclockwise or clockwise flagellar rotation, resulting in translational motion or 'tumbling', respectively. The flagellar motor is constructed from at least 20 different proteins. Although the rotor portion of the bacterial flagellar motor has been purified and studied for decades [2], there is little structural information of the stator and the export apparatus, because these membrane-associated structures are not retained following detergent extraction during the extensive basal body purification process. Recently there is a considerable interest in a thorough analysis of the structures of the periplasmic flagella $[3,4]$. In order to gain an in-depth understanding of flagellar assembly and bacterial motility, a high-throughput cryo-electron tomography system was optimized for determining 3-D structures of the intact flagellar motor from Borrelia burgdorferi [Figure 1].

Borrelia burgdorferi is a representative of highly motile and invasive bacterial pathogens: spirochetes, which cause several major diseases in humans: syphilis (Treponema pallidum), Lyme disease (Borrelia burgdorferi), and leptospirosis (Leptospira interrogans). Spirochetes have a distinctive morphology in that the flagella are enclosed within the outer membrane sheath and are thus called periplasmic flagella [5]. Because of its small cell diameter $(<0.3 \mu \mathrm{m})$, orderly arrangement of flagellar motors at each end of the bacterium, spirochetes are excellent model systems for studying the flagellar motor in living organisms.

In this study, we have developed the unique capability to obtain 3-D reconstructions of intact bacteria with unprecedented detail by using high-throughput cryo-electron tomography. By averaging $12803-$ D volumes of flagellar motors, we obtained the most detailed molecular architecture of intact flagellar motor in situ. In combination with biochemical analysis and genetic approaches, the structural location and native conformations of several key flagellar motor proteins were determined at molecular level [Figure 2]. Further analysis of the rotor/stator assembly will provide detailed information on the architecture of the intact flagellar motor, and thereby better understanding of the important process of flagellar assembly and bacterial motility.

References:

[1]. Berg HC, Annu Rev Biochem 72 (2003) 19.

[2]. Thomas DR, Francis NR, Xu C, DeRosier DJ, J Bacteriol 188 (2006) 7039.

[3]. Murphy GE, Leadbetter JR, Jensen GJ, Nature 442 (2006) 1062.

[4]. Liu J, Lin T, Botkin DJ, McCrum E, Winkler H, Norris SJ, J Bacteriol 191 (2009) 5026.

[5]. Charon NW, Goldstein SF, Annu Rev Genet 36 (2002) 47. 


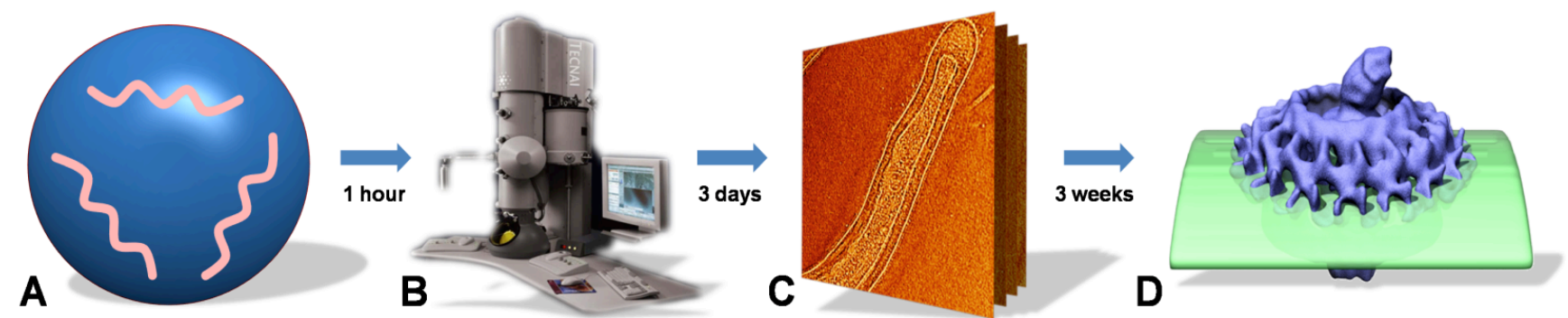

Figure 1. High-throughput pipeline of Cryo-ET: from sample to high resolution 3-D structure in situ. Preparation of frozen hydrated specimen of viable B. burgdorferi cells (A) and the loading of multiple EM grids into a TEM microscope (B) takes about one hour. Nearly 100 cryo tomography tilt series of intact cells $(\mathrm{C})$ can be generated by FEI batch tomography in three days. In three weeks, we are able to process the resulting data and determine the molecular architecture of the intact flagellar motor in situ (D).

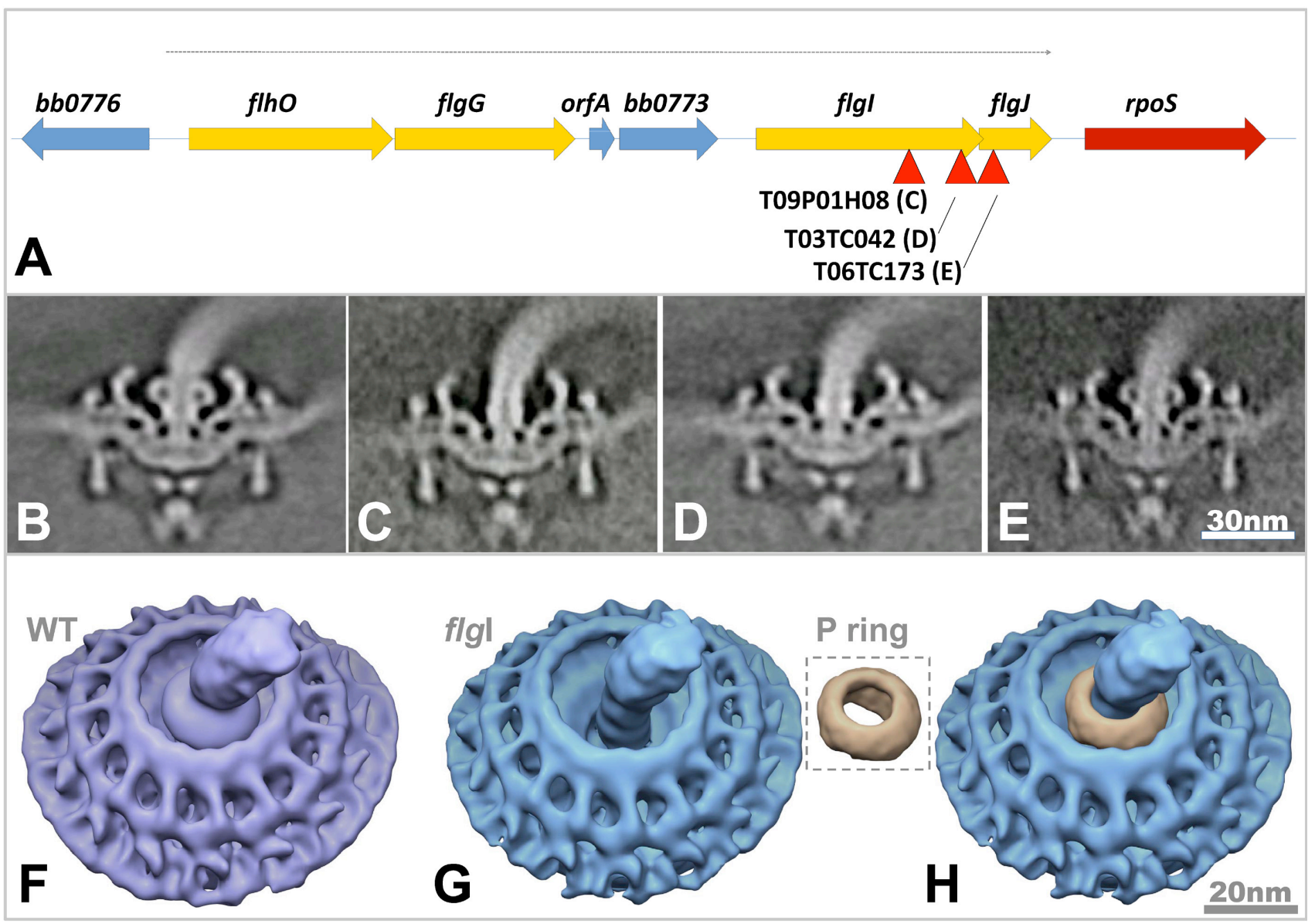

Figure 2. Localization of the $P$ ring protein Flgl in the B. burgdorferi flagellar motor, as determined by Cryo-ET derived models from transposon mutants. Panel $A$ is a map of the $B$. burgdorferi genetic locus encoding the $\mathrm{P}$ ring protein Flgl and other flagellar proteins. Gold-colored genes are flagella-related, and hypothetical genes are shown in blue. The insertion sites of three transposon mutants are shown (A). Sections through 3-D asymmetric averages of flagellar motors from WT cells (B) and three mutants T09P01H08 (C), T03TC042 (D) and T06TC173 (E) are shown. The corresponding surface renderings are from WT (F) and flgl mutant T09P01H08 ( $\mathrm{G}$ and $\mathrm{H})$ cells. The difference map between WT and the flgl mutant reveals the $P$ ring (colored beige) is shown in the inset and in the context of the motor $(\mathrm{H})$. 